\title{
Towards High-Resolution Annual Outdoor Thermal Comfort Mapping In Urban Design
}

\author{
Patrick Kastner ${ }^{1}$, Timur Dogan ${ }^{1}$ \\ ${ }^{1}$ Environmental Systems Lab, Cornell University, Ithaca, New York, USA
}

\begin{abstract}
Global warming and increasingly dense cities lead to poor outdoor thermal comfort that may not only be detrimental to our health and well-being but also decreases social and commercial activities. Although workflows for the analysis of thermal comfort exist, they have yet transitioned into the quotidian architectural design process. Our workflow allows for annual outdoor comfort analyses that are seamlessly integrated into a commonly-used CAD environment. We simulated the annual outdoor thermal comfort on a university campus and discuss which simplifications seem appropriate by means of preliminary on-site measurements. The results exemplify the possibility to conduct such analyses within reasonable time and accuracy if some simplifications to the UTCI estimation are acceptable.
\end{abstract}

\section{Introduction}

With rapid urbanization, outdoor spaces begin to compete for natural resources such as access to sun, wind, air, and daylight with other driving forces like space use efficiency. Poor outdoor thermal comfort leads to many problems related to the health and well-being of humans and also decreases social and commercial outdoor activities. Municipalities, universities, and organizations with large plots of land are making efforts toward significantly reducing their carbon footprint and focusing on sustainability. New York, for example, plans to reduce carbon emissions by 80 $\%$ by 2050 (NYC.gov, 2019) and Cornell University aims to be carbon neutral by 2030 (Collins et al., 2016). Holistic strategies are thus necessary to facilitate an urban design that fosters passively comfortable urban microclimates in light of future environmental risk and global warming.

In the last decade, the interest in outdoor thermal comfort analysis increased both in the scientific community and in practice. Some commercial tools exist which aim to simplify the input of complex physical boundary conditions by step-by-step guides. Those tools include but are not limited to ENVI-met (Huttner and Bruse, 2009), SOLWEIG (Solar and Long-Wave Environmental Irradiance Geometry (Lindberg et al., 2008), RayMan (Matzarakis et al., 2010), CitySim (Walter and Kämpf, 2015), and the Ladybug Grasshopper plugins (Mostapha Sadeghipour Roudsari, 2013). They quantify outdoor environmental conditions by estimating either the Mean Radiant Temperature (MRT) or the Universal Thermal Climate Index (UTCI). While designers, planners, and municipalities are usually committed to creating pleasant outdoor spaces, it remains difficult to assess the impact of the urban form on outdoor thermal comfort. For fast-paced urban design processes, in particular, none of the state-of-the-art approaches seem reasonable. Generating feedback about the urban design from such simulations is a computationally expensive modeling task that is cumbersome to apply in an iterative manner. Naboni et al. postulate that the acquisition of building geometry is not streamlined enough to allow for usage in an integrated architectural design environment. Hence, microclimate studies are still not widely used in the urban planning process, although studies have shown that geometric interventions are able to improve the outdoor thermal comfort in urban areas (Ebrahimabadi, 2015; Thorsson et al., 2011).

This study aims to facilitate the annual simulation of outdoor comfort and proposes an easy-to-use and reliable methodology using OpenFOAM, Radiance, and EnergyPlus to simulate the annual outdoor comfort of a university campus within a reasonable time. Further, we validate the results by means of on-site measurements and discuss possible simplifications.

\section{Case study: campus of Cornell University}

The university campus of Cornell University located in upstate NY and overlooking Lake Cayuga consists of 608 buildings and covers an area of $9.3 \mathrm{~km}^{2}$. The campus is surrounded by forest and mid-sized townhouses, see figure 1 . The section of interest in this study is the vicinity around a library building which is also used for on-site measurements, see figure $1 \mathrm{~b}$.

\section{Simulation framework for annual outdoor comfort analyses}

The simulation framework consists of several simulation engines, namely: OpenFOAM, Daysim, and Radiance, see figure 2. All engines are centered around a toolkit called Eddy $3 D$ that is implemented in Rhinoceros and Grasshopper which handles pre-, post-processing, and the data handling between those engines. We use $E d d y 3 D$ to create the simulation domain, the specification of boundary conditions, and the processing of the weather data based on the building geometry in Rhinoceros. Similarly, we use Rhinoceros' meshing capabilities to export building and terrain meshes for both OpenFOAM and Radiance. We use Radiance to simulate irradiation and compute view factors for each sensor point. From an EnergyPlus simulation, we estimate surface temperatures from which we calculate the mean radiant temperature (MRT) in combination with the respective view factor for each hour of the year and sensor point. For the annual outdoor thermal comfort evaluation, we simulated 8 wind directions in a $45^{\circ}$ interval, that serve 

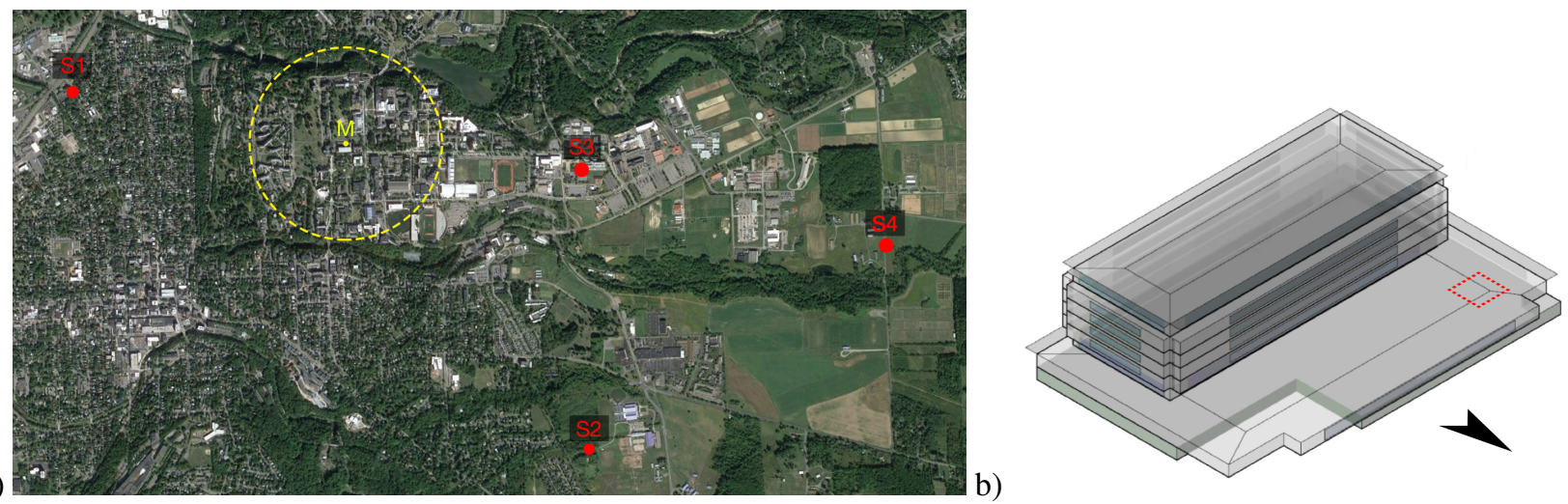

Figure 1: a) Aerial view of the university campus with the simulation domain highlighted in yellow, the library building of interest (M), and 3 local weather stations (S1-S3). Image source: Google Earth. b) 3D model of library building showing the terrace that was used for on-site measurements.

as the nearest neighbor lookup table for the annual wind velocity data. On a number of sensor points (see figure 7), we calculate the dimensionless wind velocity by dividing the probes from the CFD simulation by the scaled inlet velocity according to equation (1).

$$
U_{\text {scale }}=\frac{\frac{\kappa \cdot U}{\log \left(\frac{z_{r e f}+z_{0}}{z_{0}}\right)}}{\kappa} \cdot \log \left(\frac{r e f . h e i g h t+z_{0}}{z_{0}}\right)
$$

Then, we multiply the dimensionless wind velocity of each sensor point from the nearest simulated wind direction with the corresponding velocity and wind direction from the weather data for every hour of the year. This yields a matrix with wind reduction factors of the size $[8760 h \times$ number of sensor points] from which the wind velocities for the UTCI calculation are retrieved. Those wind velocities and mean radiant temperatures serve as input for the UTCI calculation for every hour and sensor point for which both ambient temperature and relative humidity are retrieved from the hourly weather data.

\section{Weather data}

To set up the initial simulation in a way that it facilitates later validation, seasonal analysis of historical weather data is required. We parsed weather data from the nearby Weather Underground stations KNYITHAC1 (S1), KNYITHAC52 (S2), and KNYITHAC70 (S3), and one university weather station (S4) from 2016 until 2018 - see figure 1 for their locations. We sanity-checked the data and found the same prevalent wind direction across all 4 weather stations was $\operatorname{SSE}\left(\approx 160^{\circ}\right)$ for that period. From the off-campus station S4, we extracted one day that exhibited cold/sunny weather conditions with a relatively constant wind direction for which on-campus measurements were also available. In early 2019, such conditions were present on March 27 for Ithaca, upstate NY.

\section{Universal Thermal Climate Index (UTCI)}

Like other outdoor comfort metrics such as the physiological equivalent temperature (PET), the wind chill index (WC), or the SET temperature, the UTCI was developed conceptually as an equivalent temperature measure. It is based on the multi-node thermo-physical "Fiala" model which was coupled with an adaptive clothing model to take clothing habits into account by the urban population and their behavioral changes in clothing in relation to the surrounding temperature (Fiala et al., 2012). Thus, for any combination of air temperature, wind, radiation, and humidity, UTCI is defined as the air temperature in the reference condition which would elicit the same dynamic response of the physiological model (Bröde et al., 2013). We chose the UTCI as a performance metric, as a number of studies have shown the UTCI to be a superior metric for potentially hazardous weather in winter while also achieving good performance in tropical climates (Provençal et al., 2016). Mathematically, the UTCI is a polynomial approximation that, in simple terms, may be described as (Broede, 2009; Hardy, 1998):

$$
U T C I=T_{a}+f\left(T_{a}, T_{M R T}, U, p_{\text {vapour }}\right)
$$

The resulting temperatures may then be classified and reported as thermal stress categories, see table 1. Ideally, one would strive for maximizing the annual urban climate for the "No thermal stress" condition. Undoubtedly, the $T_{M R T}$ and $U$ are the two input variables that exhibit significant spatial variation in an urban neighborhood, whereas $T_{a}$ and $p_{\text {vapour }}$ remain relatively spatially constant.

Table 1: Thermal stress categories of the UTCI.

\begin{tabular}{ll}
\hline UTCI $\left[{ }^{\circ} \mathrm{C}\right]$ & Stress category \\
\hline$>46$ & Extreme heat stress \\
+38 to +46 & Very strong heat stress \\
+32 to +38 & Strong heat stress \\
+26 to +32 & Moderate heat stress \\
+9 to +26 & No thermal stress \\
+9 to 0 & Slight cold stress \\
0 to -13 & Moderate cold stress \\
-13 to -27 & Strong cold stress \\
-27 to -40 & Very strong cold stress \\
$<-40$ & Extreme cold stress \\
\hline
\end{tabular}




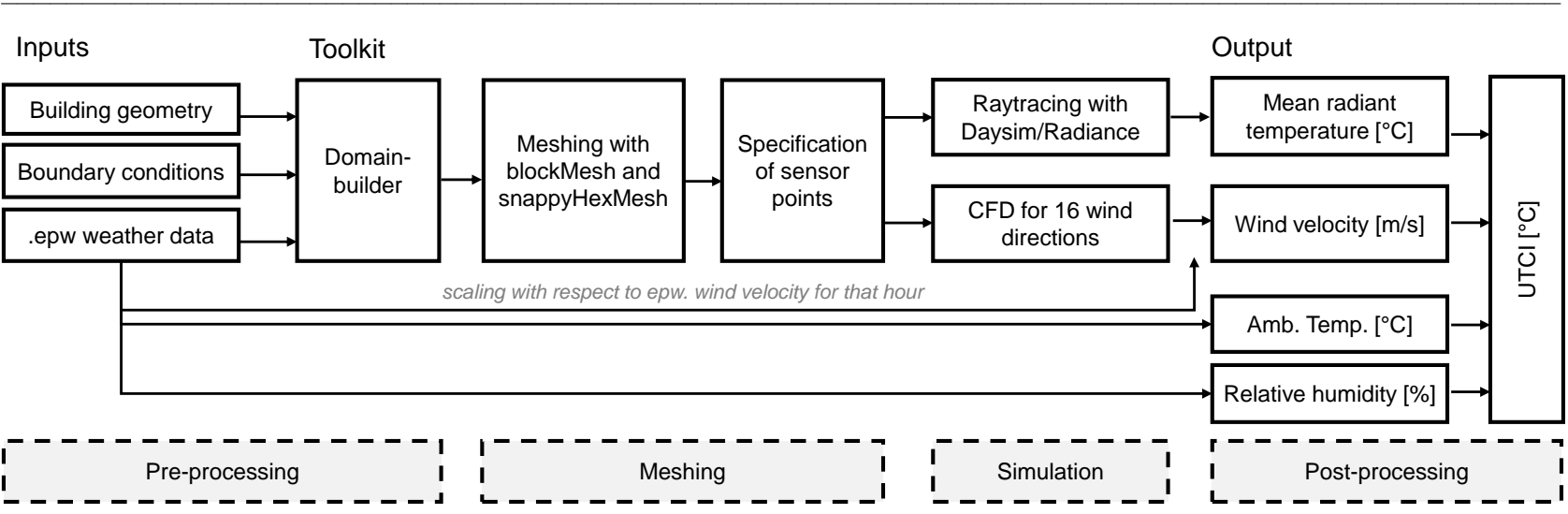

Figure 2: Flow chart that depicts the pre-processing, simulation, and post-processing workflows developed in this study.

\section{CFD simulations}

\section{Computational model and simulation domain}

Ithaca's elevation varies to the extent that it is necessary to make use of a digital elevation model. The model we used incorporates the building footprints which we extruded to their actual height reported in the campus GIS repository.

\section{Computational mesh}

The mesh was created by OpenFOAM's blockMesh utility for the background mesh and snappyHexMesh to subsequently snap the background mesh to the building geometry. For the background mesh, we used a cylindrical simulation domain approach discussed in (Kastner and Dogan, 2018). This meshing approach allows for reusing the same computational mesh for every subsequent wind direction. The simulation domain was set up with a radius of $500 \mathrm{~m}$ around the library building and a height of $350 \mathrm{~m}$ while taking into account all relevant surrounding buildings which resulted in $11.6 \times 10^{6}$ cells. A side view of the cylindrical mesh with the terrain cutout is given in figure 3 .

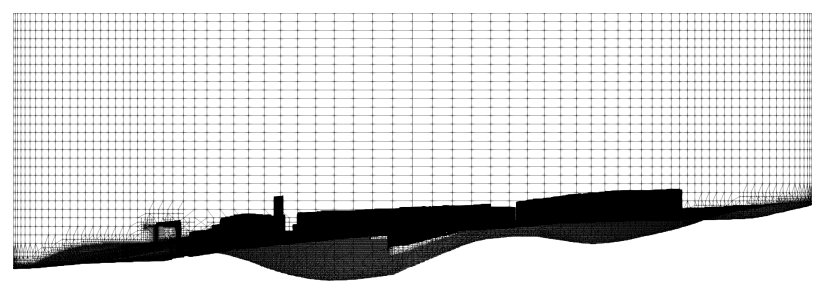

Figure 3: Side view of mesh with terrain cutout and the silhouette of the campus buildings.

\section{Boundary conditions}

The Grasshopper plugin called $E d d y 3 D$ was used to automate the pre-processing, including the assignment of boundary conditions. Depending on the wind direction, we mapped the inlets to a one-half circle of the simulation domain and the outlet on the opposite side as described in (Kastner and Dogan, 2018). The half circular domain inlet was set to a uniform profile for $U, k$, and $\omega$, and a roughness length $z_{0}=1$ that corresponds to "regular coverage with large size obstacles with open spaces roughly equal to obstacle heights, suburban houses" (Wallace and Hobbs, 2006), according to equations 3-5. The particular wind direction used for the validation study was $310^{\circ}$, based on the measured weather data. At the outlet of the computational domain, constant pressure is assumed, while the other variables are imposed to be zero-gradient. The ground and the building geometry use the same boundary conditions, a no-slip condition for velocity, a zero-gradient condition for the pressure and wall functions for $k$ and $\omega$. For the field turbulence eddy viscosity $v_{t}$, the intelligent wall function called nutUSpaldingWallFunction was used, given its universal applicability across wide ranges of $y^{+}$ values (De Villiers, 2006). The front, back, and top faces are set to a symmetry boundary conditions for all variables. The kinematic viscosity, $v$, was set to $1.5 \times 10^{-5}$. The turbulence inlet parameters were calculated using the following equations:

$$
\begin{aligned}
k & =1.5 \cdot T_{u}^{2} \cdot U_{r e f}^{2} \\
\varepsilon & =\frac{C_{m u} \cdot k^{2}}{v \cdot \frac{\mu_{t}}{\mu}} \\
\omega & =\frac{\varepsilon}{C_{m u} \cdot k}
\end{aligned}
$$

\section{Other computational parameters}

Since CFD is currently a bottleneck in this simulation procedure, we used an incompressible, isothermal, steadystate solver from OpenFOAM in combination with a $k-\omega-S S T$ RANS turbulence model to calculate the wind velocities that are needed for the UTCI calculation. We chose the $k-\omega-S S T$ based on its superior accuracy while only being slightly more computationally expensive (Ramponi and Blocken, 2012). The pressure-velocity coupling was established with the SIMPLE algorithm using three non-orthogonal correctors. Buoyancy effects were neglected due to air velocities that are well above $1.8 \mathrm{~m} \mathrm{~s}^{-1}$ (Tecle et al., 2013; Boulard et al., 1996; Magnusson et al., 
2014). We ran all the simulations for 10000 iterations, for which the simulations reached the following residuals: $1 \times 10^{-4}$ for $\omega, 1 \times 10^{-4}$ for $k, 5 \times 10^{-2}$ for $p, 1 \times 10^{-4}$ for $U_{x}, U_{z}$, and $U_{y}$. The relaxation factors were chosen to be 0.3 for $p$ and 0.7 for $U, k$ and $\omega$. All simulations ran on an AMD Ryzen Threadripper 1950X 16-Core Processor running Windows 10. We used the Docker Version 2.0.1.0 (30090) to run OpenFOAM 4.1.

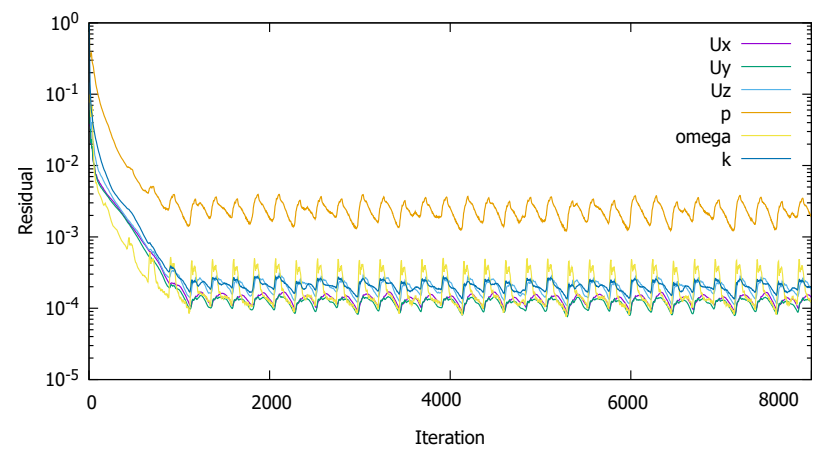

Figure 4: Residuals of the CFD simulation.

\section{MRT calculation}

Studies have analyzed several methods to estimate the MRT that range from simple curve fits more holistic view factor analysis methods (Kessling et al., 2013; Thorsson et al., 2007). In this study, the MRT calculation was conducted with the help of EnergyPlus with which we calculated exterior surface temperatures. We then used Radiance to calculate view factors with respect to all building surfaces, ground patches and sky for all sensor points. From those values, we calculated the solar-adjusted mean radiant temperature according to:

$$
M R T=\left[\left(c_{s} \cdot \alpha_{s} \cdot I \cdot \frac{1}{\sigma}\right)+\sum_{i=1}^{n_{v f}} F_{p, i} \cdot T_{s r f, i}^{4}\right]^{\frac{1}{4}}-273
$$

where $M R T$ is Mean Radiant Temperature in ${ }^{\circ} \mathrm{C}, I$ is the hourly irradiation in $\mathrm{W} \mathrm{m}^{-2}, \sigma$ is the Stefan-Boltzmann constant, $c_{s}=0.25$ is a projection factor assumed for seating, $\alpha_{s}=0.7$ is an assumed skin absorption coefficient, $F_{p, i}$ is the view factor between a sensor point and a surface " $i$ ", $T_{s r f, i}$ is the surface temperature in $K$.

\section{Preliminary validation with on-site measure- ments}

Validation with on-site measurements is essential for a complex metric with multiple inputs such as the UTCI. To validate simulation results, we meter weather data including temperature, humidity, and radiation as well as wind speed and direction at an undisturbed and well-exposed location ca. $1 \mathrm{~km}$ from campus, see $\mathrm{S} 4$ in figure 1 . In parallel, we monitor relevant UTCI components such as $T_{a m b}, M R T$, wind speed and radiation exposure at the observation point shown in figure 6, using a Thermal Micro Climate Data Logger shown figure 5. We then translate the raw weather data into the EPW format utilizing psychometric calculations and an irradiation split into indirect and diffuse solar radiation using the HDKR/Reindl model (Reindl and Beckman, 1990). This EPW file serves as weather input for the simulation model that we used to approximate UTCI parameters for later comparison against measurements at the observation point. The measurements were taken on the paved terrace of the library building to minimize the influence of evaporative cooling in this area, which is not considered in our model, see figure 5. The measurement frequencies were set to $15 \mathrm{~s}$ which was averaged with a moving average over 240 instances. During the measurements, the approaching velocity at S4 was observed, in order to wait for periods of time in which constant 60-minute averaged wind speeds and directions were present, which was the case between 13:00 and 16:00 $\mathrm{h}$ on March 27, see table 2. Here, we treated winds to come from a "constant" direction if they lie within a $22^{\circ}$ angle sector. With those 60-minute intervals, we were able to extract time windows that were used as inputs for our simulations. In sum, we used wind velocity, wind direction, and radiation measurements from the undisturbed, unshaded weather station as input for our simulation whereas ambient temperature, and relative humidity are taken from the measurements on site.

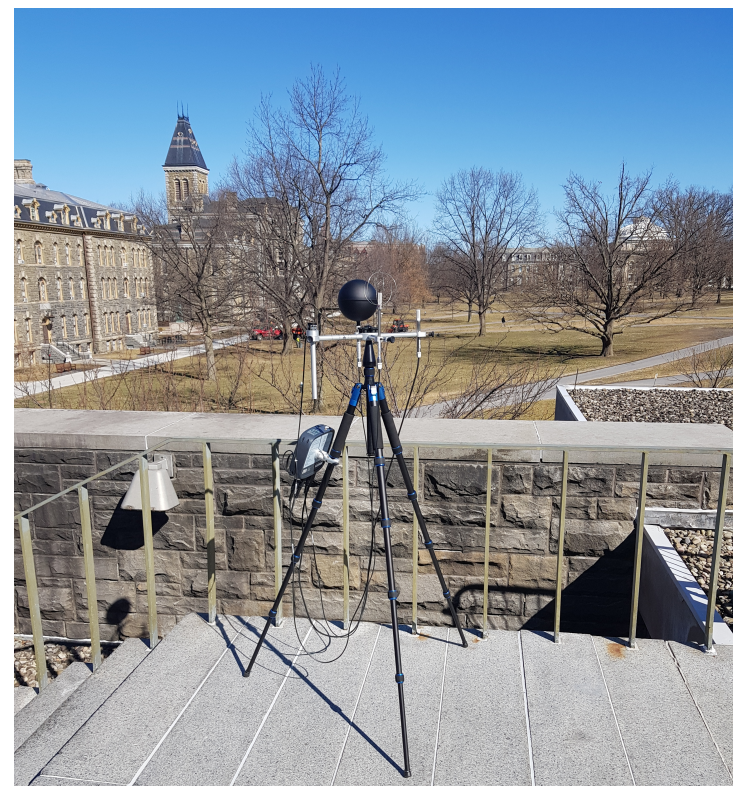

Figure 5: HD32.1 - Thermal Microclimate Data Logger used as measurement setup with sensors for ambient temperature, relative humidity, global irradiation, and globe thermometer. The photo was taken in the direction of the approaching flow.

\section{Results}

With respect to validation of the CFD results, we report that the moving average of the wind velocity at the measurement location yields $2.5 \mathrm{~m} \mathrm{~s}^{-1}$. The average probed velocity from the red rectangle in figure 6 shows a value of $2.6 \mathrm{~m} \mathrm{~s}^{-1}$, probing the larger rectangle yields $3.2 \mathrm{~m} \mathrm{~s}^{-1}$. 
Table 2: Hourly average off-site measurements from the weather station $S 4$ on March 27, 2019. The on-site measurements shown in figure 6 were taken from 10:15 h to 18:30 h. From 13:00 to 16:00 h, both wind velocity and direction were fairly constant. The wind direction $310^{\circ}$ was later used for the validation study.

\begin{tabular}{ccccccccc}
\hline \multirow{2}{*}{ Hour } & \multicolumn{2}{c}{ Air temp $\left[{ }^{\circ} \mathrm{C}\right]$} & RH [\%] & \multicolumn{2}{c}{ Wind speed $(\mathbf{m} / \mathbf{s})$} & \multicolumn{2}{c}{ Wind direction } \\
Max & Avg & & Max & Avg & deg & comp & Irradiation $\left[W / m^{2}\right]$ \\
\hline 18:00 & 2.8 & 1.7 & 32 & 6.9 & 4.2 & 341 & NNW & 277 \\
17:00 & 2.8 & 2.2 & 34 & 8.4 & 4.6 & 321 & NW & 447 \\
$16: 00$ & 2.2 & 1.7 & 32 & 8.7 & 5.1 & 315 & NW & 599 \\
$15: 00$ & 2.2 & 1.1 & 35 & 9.0 & 5.1 & 304 & NW & 704 \\
$14: 00$ & 1.1 & 0.6 & 39 & 7.9 & 4.9 & 307 & NW & 748 \\
$13: 00$ & 1.1 & 0.0 & 42 & 8.1 & 4.8 & 317 & NW & 741 \\
$12: 00$ & 0.6 & 0.0 & 42 & 6.8 & 4.2 & 326 & NNW & 668 \\
\hline
\end{tabular}

Figure $7 \mathrm{a}-\mathrm{b}$ ) show a top view of the campus with UTCI map for cold and sunny weather conditions recorded on March 27, 2019, by the station S4, assuming a constant wind velocity from $310^{\circ}(\mathrm{NW})$. We can see that the overall UTCI patterns follow the daily cycle prescribed by the ambient temperature, which increases from 12:00 $\mathrm{h}-16: 00$ $\mathrm{h}$. It is, further, evident that campus buildings provide shelter against the wind, resulting in a UTCI increase in their wake region. Finally, Figure 7 c) depicts the annual percentage of hours within comfort (without thermal stress) across campus.

\section{a) Top-view of cropped CFD result}

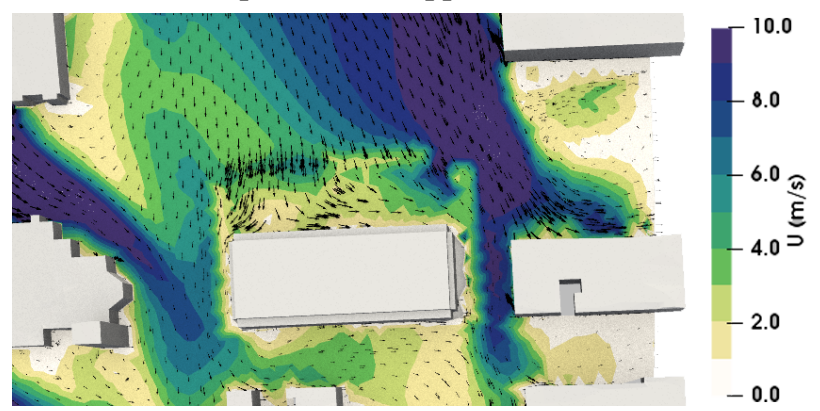

b) Probed velocities around the measurement location

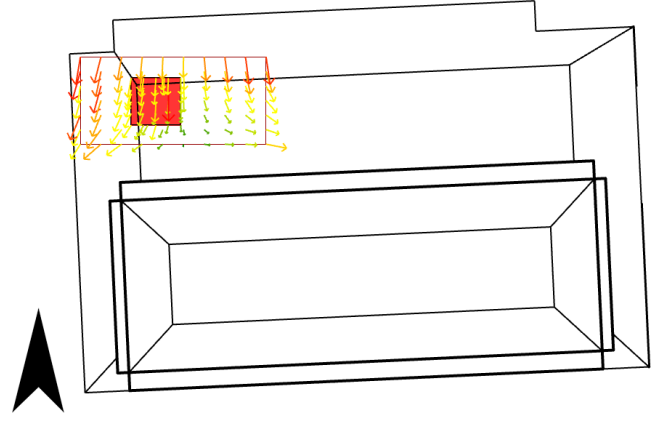

Figure 6: Measurement location for the UTCI validation highlighted in red in the figure at the top. a) Top-view of cropped CFD result at $2 \mathrm{~m}$ above ground. $b$ ) The average wind velocity probed from the red rectangle at the measurement location yields $2.6 \mathrm{~m} \mathrm{~s}^{-1}$; the average wind velocity probed from the larger rectangle around the measurement location yields $3.2 \mathrm{~m} \mathrm{~s}^{-1}$.

\section{Discussion}

\section{General limitations}

The results presented show that it is possible to simulate the annual outdoor comfort in an urban environment with reasonable effort. The simplified methodology presented, however, comes with limitations with respect to its general applicability.

First, this study relies on separately validated engines as opposed to one integrated engine such as for example ENVImet or CitySim. As the UTCI is a metric that is derived from multiple input data, the accuracy of these simulation results depends on both the accuracy of every individual engine and the extent of transient behavior occurring in the real world. In the interest of simulation time, the simulation engines in this paper were linked by static, external, one-dimensional coupling, see figure 2 , which is the simplest of the three possible coupling strategies (Barbason and Reiter, 2014; Odnevall Wallinder et al., 2002). This brings both advantages and disadvantages. For one, every simulation engine has been validated on its own and there exists trust among the research community that Radiance, EnergyPlus and OpenFOAM are in principle able to generate accurate results. What is not possible, however, is the reverse data exchange due to two major differences between the approaches, namely the temporal difference of simulated time (months to years for BES vs. hours calculated per time step in seconds for CFD) and the difference in computation time. As a result, no transient behavior across the individual engines, such as thermal mass being in an energy exchange relation through convective heat transfer, is taken into account by this approach. It follows that the more transient behavior the system exhibits, the less accurate the presented model tends to be.

Second, the RANS CFD methodology we use in this study is known to have flaws when it comes to predicting turbulent behavior in wake regions of buildings. Further, the amount of wind buffeting that can be modeled with more advanced methods like LES cannot be estimated due to the averaging nature of the RANS model. At the same time, the sensitivity of the wind velocity for arbitrary UTCI values increases with decreasing ambient temperature. In other words, the colder the ambient temperature, the more dominant the wind chill effect due to convective heat transfer 
a) $14: 00 \mathrm{~h}$

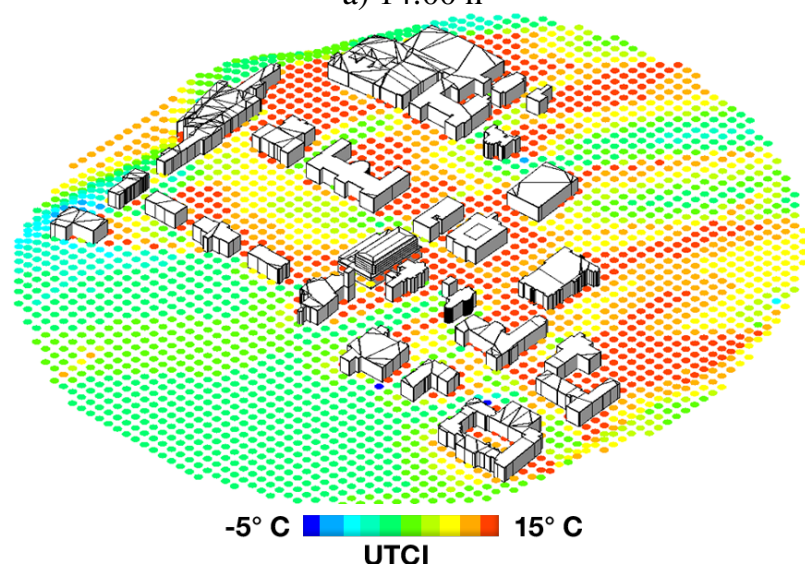

b) $16: 00 \mathrm{~h}$

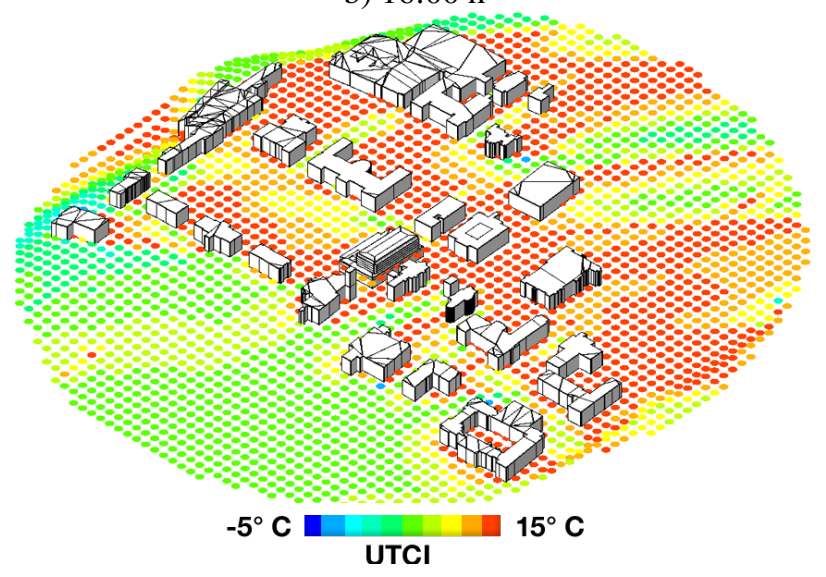

c) Annual hours within thermal comfort

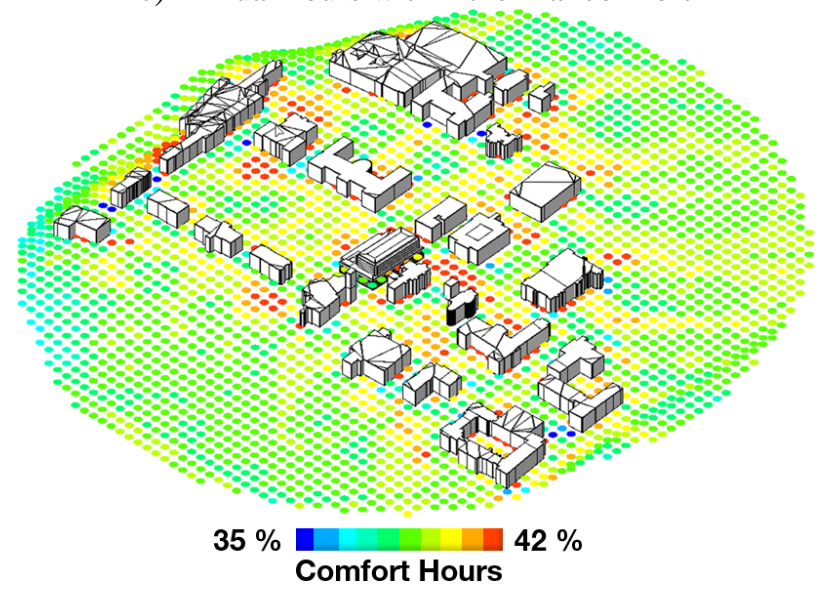

Figure 7: UTCI and comfort hour maps. $a-b)$ Shows the simulated UTCI distribution of the campus on March 27, 2019, for 14:00 hand 16:00 h. c) Shows the calculated percentage of annual thermal comfort hours with "no thermal stress", see table 1.

will be (Bröde et al., 2013). Consequently, when climates with low ambient temperatures are studied, one should keep in mind that such effects are not taken into account and appropriate factors of safety should be considered. Mackey et al. conducted extensive studies on the sensibility of input variables for microclimate studies. They found that the influence of the number of wind directions simulated is almost negligible for a tropical climate such as that in Singapore. While this seems plausible for a tropical climate, it is worth reiterating that microclimate maps will likely suffer greatly from a reduced number of simulated wind directions in colder climates (Bröde et al., 2013). Although additional wind directions increase the simulation time for the engine that is already the bottleneck in simulation procedure, the RANS method is state-of-the-art in terms of an accuracy/efficiency/robustness-trade-off for outdoor comfort mappings, especially when additional measures are taken into account (Kastner and Dogan, 2018). However, one area for further research might be to replace the RANS method with an engine that is based on Lattice Boltzmann methods which can be solved less expensively.

The comparison between the CFD simulation and the one measurement location shows a fairly good agreement. We, however, concede, that a single reference point in the upwind region of a building is not sufficient for a holistic validation of the presented method. Further, we probed and average from a larger area in front of the building the deviation between both increases from $10 \%$ to $22 \%$. This clearly shows how highly sensitive the measurement location is, which in turn shows the necessity for a higher number of measurements for future studies both in terms of the number of measurement locations themselves but also the length and number of measurements for each location over time.

\section{Overall accuracy vs. level of detail necessary during design process}

There are unanswered questions with respect to microclimate maps that are created during the design process, compared to maps that analyze an already existing urban environment. Those questions usually revolve around a trade-off between accuracy and computational efficiency.

First, the method to estimate the MRT in a reasonable time without relying on the knowledge of all materials and their corresponding emissivities is not yet agreed upon. The overall goal for a tool such as the one presented is computational efficiency as it ought to be used during the design process. Thus, one might argue that it is sufficient if only the thermal stress categories are correctly predicted for a sufficiently high percentage of hours throughout the year as those ultimately constitute the optimization goal for the urban area, see table 1. To claim such accuracy, validation studies for more hours in a year will have to be carried out.

Second, how and when should evaporative cooling be considered without introducing a host of input uncertainties with respect to e.g. plants, rainfall, soil composition, and pavement area? Integrated simulation tools such as ENVI-met are capable of integrating evaporative cooling by means of internal coupling. Such modeling approaches require detailed knowledge about the additional simulation input, namely the rainfall frequency, the type of plants and trees to be used, their porosity, and the soil composition (Manickathan et al., 2018). Unfortunately, in commonly used EPW weather files there is rarely any rain data avail- 
able, and if there is, its validity is questionable with respect to a "typical" amount of rainfall per year. In case of a lack of high-quality input data, the majority of inputs would thus have to be substituted by default values which could be counterproductive for the desired information gain. Regarding the external coupling used in our approach, it is also questionable if incorporating vegetation is feasible as it would pose great challenges on the data exchange necessary due to shading by trees and the benefits from evaporative cooling - the former of which has been shown to be more beneficial by Manickathan et al..

Aside from that, Mackey et al. found that the sky heat exchange is the most dominant variable in outdoor comfort modeling, followed by wind patterns, and UHI/surface temperature, producing errors that range from $2.4-0.5^{\circ} \mathrm{C}$ respectively. Although EP's simulation time currently does not constitute the bottleneck, one might argue in favor of simplifying the MRT calculations by using an admittance method or an even simpler linear regression going forward (M.G, 1994; Kessling et al., 2013).

We neglected buoyancy on the CFD side not only to gain simplicity in selecting the boundary conditions but also because it is less computationally expensive. As shown in the validation study, this could be the reason for a large discrepancy between the model and the measurements. Allegrini and Carmeliet showed a correlation between the local air temperature, the volumetric flow rates in predefined control volumes, and the local thermal diffusivity in those volumes. In future studies of urban areas, this approach could be used to predict the local heat island risk of urban environments while utilizing an isothermal CFD approach. Going forward, we hope to be able to define a threshold for which the predicted air temperature from that method would be used as an input for the UTCI.

\section{Summary and conclusions}

The authors implemented an outdoor comfort modeling framework for urban design in $\mathrm{C \#}$ as a plugin for Rhinoceros and Grasshopper that enables seamless workflow integration. UTCI simulation results around a library building on a university campus are available and seem plausible. The results exemplify the possibility to conduct such analyses within reasonable time and accuracy if some simplifications to the UTCI estimation are acceptable. In the future, we plan to increase the number of measurement locations to be able to present a more holistic validation of our simulation results.

\section{Acknowledgements}

The authors would like to thank the David R. Atkinson Center for a Sustainable Future and The Center for Transportation, Environment, and Community Health $(\mathrm{CTECH})$ for funding this research.

\section{References}

Allegrini, J. and J. Carmeliet (2017). Coupled cfd and building energy simulations for studying the impacts of building height topology and buoyancy on local urban microclimates. Urban Climate 21, 278-305.

Barbason, M. and S. Reiter (2014). Coupling building energy simulation and computational fluid dynamics: Application to a two-storey house in a temperate climate. Building and Environment 75, 30-39.

Boulard, T., J. Meneses, M. Mermier, and G. Papadakis (1996). The mechanisms involved in the natural ventilation of greenhouses. Agricultural and Forest Meteorology 79(1-2), 61-77.

Bröde, P., K. Błazejczyk, D. Fiala, G. Havenith, I. Holmér, G. Jendritzky, K. Kuklane, and B. Kampmann (2013, feb). The Universal Thermal Climate Index UTCI compared to ergonomics standards for assessing the thermal environment. Industrial health 51(1), 16-24.

Leibniz Research Centre for Working Environment and Human Factors (2009). The Universal Thermal Climate Index.

Cornell University (2016). Options for Achieving a Carbon Neutral Campus by 2035.

De Villiers, E. (2006). The potential of large eddy simulation for the modeling of wall bounded flows. Ph. D. thesis, Imperial College of Science, Technology and Medicine.

Ebrahimabadi, S. (2015). Outdoor Comfort in Cold Climates: Integrating Microclimate Factors in Urban Design. $\mathrm{Ph}$. D. thesis, Luleå tekniska universitet.

Fiala, D., G. Havenith, P. Bröde, B. Kampmann, and G. Jendritzky (2012). UTCI-Fiala multi-node model of human heat transfer and temperature regulation. International Journal of Biometeorology 56(3), 429-441.

Hardy, B. (1998). Its-90 formulations for vapor pressure, frostpoint temperature, dewpoint temperature, and enhancement factors in the range-100 to+ $100 \mathrm{c}$. In Proceedings of the third international symposium on humidity and moisture, Teddington, London, England.

Huttner, S. and M. Bruse (2009). Numerical modeling of the urban climate-a preview on envi-met 4.0. In 7th International Conference on Urban Climate ICUC-7, Yokohama, Japan, Volume 29.

Kastner, P. and T. Dogan (2018). Streamlining meshing methodologies for annual urban CFD simulations. In eSim 2018.

Kessling, W., M. Engelhardt, and D. Kiehlmann (2013). The human bio-meteorological chart. In PLEA.

Lindberg, F., B. Holmer, and S. Thorsson (2008). Solweig $1.0-$ modelling spatial variations of $3 \mathrm{~d}$ radiant fluxes and mean radiant temperature in complex urban settings. International journal of biometeorology 52(7), 697-713. 
Mackey, C., T. Galanos, L. Norford, M. S. Roudsari, and N. S. Bhd (2017). Wind, Sun, Surface Temperature, and Heat Island : Critical Variables for High-Resolution Outdoor Thermal Comfort. In Proceedings of the 15th IBPSA Conference San Francisco.

Magnusson, S., A. Dallman, D. Entekhabi, R. Britter, H. J. Fernando, and L. Norford (2014). On thermally forced flows in urban street canyons. Environmental Fluid Mechanics 14(6), 1427-1441.

Manickathan, L., T. Defraeye, J. Allegrini, D. Derome, and J. Carmeliet (2018). Parametric study of the influence of environmental factors and tree properties on the transpirative cooling effect of trees. Agricultural and Forest Meteorology 248(October 2017), 259-274.

Matzarakis, A., F. Rutz, and H. Mayer (2010). Modelling radiation fluxes in simple and complex environments: basics of the rayman model. International journal of biometeorology 54(2), 131-139.

M.G, D. (1994). The thermal response of an enclosure to periodic excitation: The CIBSE approach. Building and Environment 29(2), 217-235.

Mostapha Sadeghipour Roudsari, M. P. (2013). LADYBUG: A PARAMETRIC ENVIRONMENTAL PLUGIN FOR GRASSHOPPER TO HELP DESIGNERS CREATE AN ENVIRONMENTALLY-CONSCIOUS DESIGN. In 13th Conference of International Building Performance Simulation Association, pp. 3128-3135.

Naboni, E., S. Coccolo, M. Meloni, and J.-L. Scartezzini (2018). OUTDOOR COMFORT SIMULATION OF COMPLEX ARCHITECTURAL DESIGNS A REVIEW OF SIMULATION TOOLS FROM THE DESIGNER PERSPECTIVE. In 2018 Building Performance Analysis Conference and SimBuild, pp. 659-666.

NYC.gov (2019). The new york city carbon challenge 2019.

Odnevall Wallinder, I., J. Lu, S. Bertling, and C. Leygraf (2002). On approaches to couple energy simulation and computational fluid dynamics programs. Building and Environment 37(8-9), 857-864.

Provençal, S., O. Bergeron, R. Leduc, and N. Barrette (2016). Thermal comfort in Quebec City, Canada: sensitivity analysis of the UTCI and other popular thermal comfort indices in a mid-latitude continental city. International Journal of Biometeorology 60(4), 591-603.

Ramponi, R. and B. Blocken (2012). Cfd simulation of cross-ventilation for a generic isolated building: impact of computational parameters. Building and Environment 53, 34-48.

Reindl, D. and W. Beckman (1990). Diffuse fraction correlations. Solar Energy 45(1), 1-7.
Tecle, A., G. T. Bitsuamlak, and T. E. Jiru (2013). Winddriven natural ventilation in a low-rise building: A Boundary Layer Wind Tunnel study. Building and Environment 59, 275-289.

Thorsson, S., F. Lindberg, J. Björklund, B. Holmer, and D. Rayner (2011). Potential changes in outdoor thermal comfort conditions in gothenburg, sweden due to climate change: the influence of urban geometry. International Journal of Climatology 31(2), 324-335.

Thorsson, S., F. Lindberg, I. Eliasson, and B. Holmer (2007). Different methods for estimating the mean radiant temperature in an outdoor urban setting. International journal of climatology 27(14), 1983-1993.

Wallace, J. M. and P. V. Hobbs (2006). Atmospheric Science: An Introductory Survey (2 ed.). Academic Press.

Walter, E. and J. H. Kämpf (2015). A verification of CitySim results using the BESTEST and monitored consumption values. In 2nd Building Simulation Applications conference, pp. 215-222.

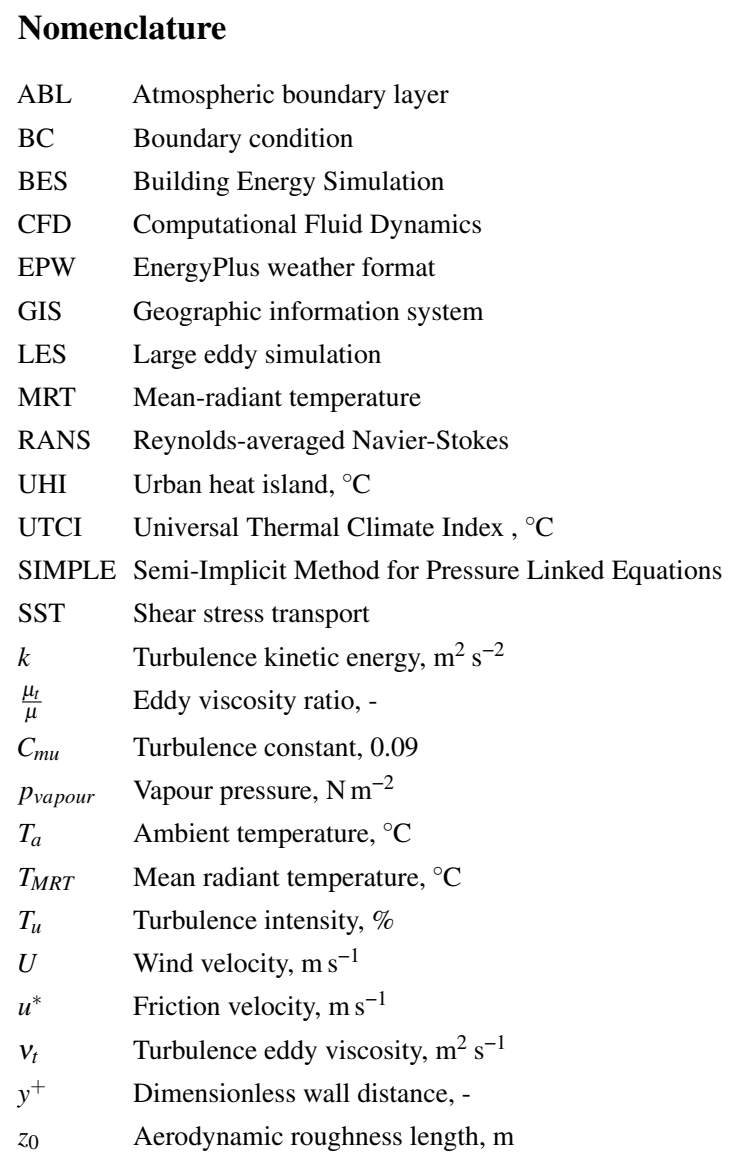

